\title{
Contact Allergy to Topical Medicaments, Part 1: A Double-edged Sword
}

\author{
Ashley Ng, BS; Amber Reck Atwater, MD; Margo Reeder, MD
}

\section{PRACTICE POINTS}

- Allergic contact dermatitis should be suspected in patients with persistent or worsening dermatitis after use of topical medications.

- Prior sensitization is not always apparent, and cross-reactions may occur between structurally similar compounds.

- Although most medicaments can be patch tested as is, patch testing to the individual components may be necessary to identify the causative allergen.

Topical drugs are used to treat a variety of cutaneous and noncutaneous conditions. Direct application to the skin can result in adverse cutaneous effects, including allergic contact dermatitis (ACD). In this article, we review medicament ACD with a focus on acne and rosacea medications, antimicrobials, antihistamines, and topical pain preparations.

Cutis. 2021;108:271-275.

7 opical medications frequently are prescribed in dermatology and provide the advantages of direct skin penetration and targeted application while typically sparing patients from systemic effects. Adverse cutaneous effects include allergic contact dermatitis (ACD), irritant contact dermatitis (ICD), photosensitivity, urticaria, hyperpigmentation or hypopigmentation, atrophy, periorificial dermatitis, and acneform eruptions. Allergic contact dermatitis can develop from the active drug or vehicle components.

Patients with medicament ACD often present with symptoms of pruritus and dermatitis at the site of topical application. They may express concern that the medication is no longer working or seems to be making things worse. Certain sites are more prone to developing medicament dermatitis, including the face, groin, and lower legs. Older adults may be more at risk. Other risk factors include pre-existing skin diseases such as stasis dermatitis, acne, psoriasis, atopic dermatitis, and genital dermatoses. ${ }^{1}$ A review of 14,911 patch-tested patients from a single referral clinic revealed that $17.4 \%$ had iatrogenic contact dermatitis, with the most common culprits being topical antibiotics, antiseptics, and steroids. ${ }^{2}$

In this 2-part series, we will focus on the active drug as a source of ACD. Part 1 explores ACD associated with acne and rosacea medications, antimicrobials, antihistamines, and topical pain preparations.

\section{Acne and Rosacea Medications}

Retinoids-Topical retinoids are first-line acne treatments that help normalize skin keratinization. Irritant contact dermatitis from retinoids is a well-known and common side effect. Although far less common than ICD, ACD from topical retinoid use has been reported., Reactions to tretinoin are most frequently reported in the literature compared to adapalene gel ${ }^{5}$ and tazarotene foam, which have lower potential for sensitization. ${ }^{6}$ Allergic contact dermatitis also has been reported from retinyl palmitate ${ }^{7,8}$ in cosmetic creams and from occupational exposure in settings of industrial vitamin A production. ${ }^{9}$ Both ICD and ACD from topical retinoids can present with pruritus, erythema, and scaling. Given this clinical overlap between ACD and ICD, patch testing is crucial in differentiating the underlying etiology of the dermatitis.

Benzoyl Peroxide-Benzoyl peroxide (BP) is another popular topical acne treatment that targets Cutibacterium acnes, a bacterium often implicated in the pathogenesis of acne

Ms. Ng and Dr. Reeder are from the Department of Dermatology, University of Wisconsin School of Medicine and Public Health, Madison. Dr. Atwater is from the Department of Dermatology, Duke University School of Medicine, Durham, North Carolina, and Eli Lilly and Company, Indianapolis, Indiana.

Ms. Ng and Dr. Reeder report no conflict of interest. Dr. Atwater is Immediate Past President of the American Contact Dermatitis Society (ACDS) and is an employee of Eli Lilly and Company.

This article is the first of a 2-part series. Part 2 will appear in January 2022.

Correspondence: Margo Reeder, MD, 1 S Park St, 7th FI, Madison, WI 53715 (mreeder@dermatology.wisc.edu).

doi:10.12788/cutis.0390 
vulgaris. Similar to retinoids, ICD is more common than ACD. Several cases of ACD to BP have been reported. ${ }^{10-14}$ Occasionally, honey-colored crusting associated with ACD to $\mathrm{BP}$ can mimic impetigo. ${ }^{10}$ Aside from use of $\mathrm{BP}$ as an acne treatment, other potential exposures to BP include bleached flour ${ }^{13}$ and orthopedic bone cement. Occupations at risk for potential BP exposure include dental technicians ${ }^{15}$ and those working in plastic manufacturing.

Brimonidine-Brimonidine tartrate is a selective $\alpha_{2}$-adrenergic agonist initially used to treat open-angle glaucoma and also is used as a topical treatment for rosacea. Allergic reactions to brimonidine eye drops may present with periorbital hyperpigmentation and pruritic bullous lesions. ${ }^{16}$ Case reports of topical brimonidine ACD have demonstrated mixed patch test results, with positive patch tests to Mirvaso (Galderma) as is but negative patch tests to pure brimonidine tartrate $0.33 \% .{ }^{17,18}$ Ringuet and Houle ${ }^{19}$ reported the first known positive patch test reaction to pure topical brimonidine, testing with brimonidine tartrate $1 \%$ in petrolatum. ${ }^{20,21}$ Clinicians should be attuned to ACD to topical brimonidine in patients previously treated for glaucoma, as prior use of ophthalmic preparations may result in sensitization. ${ }^{18,20}$

\section{Antimicrobials}

Clindamycin-Clindamycin targets bacterial protein synthesis and is an effective adjunct in the treatment of acne. Despite its widespread and often long-term use, topical clindamycin is a weak sensitizer. ${ }^{22}$ To date, limited case reports on $\mathrm{ACD}$ to topical clindamycin exist. ${ }^{23-28}$ Rare clinical patterns of ACD to clindamycin include mimickers of irritant retinoid dermatitis, erythema multiforme, or pustular rosacea..$^{25,26,29}$

Metronidazole-Metronidazole is a bactericidal agent that disrupts nucleic acid synthesis with additional antiinflammatory properties used in the treatment of rosacea. Allergic contact dermatitis to topical metronidazole has been reported. ${ }^{30-34}$ In 2006, Beutner at $\mathrm{al}^{35}$ patch tested 215 patients using metronidazole gel 1\%, which revealed no positive reactions to indicate contact sensitization. Similarly, Jappe et $\mathrm{al}^{36}$ found no positive reactions to metronidazole $2 \%$ in petrolatum in their prospective analysis of 78 rosacea patients, further highlighting the exceptionally low incidence of ACD. Cross-reaction with isothiazolinone, which shares structurally similar properties to metronidazole, has been speculated. ${ }^{31,34}$ One patient developed an acute reaction to metronidazole gel 0.75\% within 24 hours of application, suggesting that isothiazolinone may act as a sensitizer, though this relationship has not been proven. ${ }^{31}$

Neomycin-Neomycin blocks bacterial protein synthesis and is available in both prescription and overthe-counter (OTC) formulations. It commonly is used to treat and prevent superficial wound infections as an OTC antibiotic and also has otic, ophthalmologic, gastroenterologic, urologic, and peritoneal formulations. It also can be used in the dental and veterinary fields and is present in some animal feeds and in trace amounts in some vaccines for humans. Neomycin is a common antibiotic contact allergen, and the most recently reported 2017-2018 North American Contact Dermatitis Group data cycle placed it at number 12 with $5.4 \%$ positivity. ${ }^{37}$ Co-reactions with bacitracin can occur, substantially limiting OTC topical antibiotic options for allergic patients. A safe alternative for patients with neomycin (and bacitracin and polymyxin) contact allergy is prescription mupirocin.

Bacitracin-Bacitracin interferes with peptidoglycan and cell-wall synthesis to treat superficial cutaneous infections. Similar to neomycin, it also can be found in OTC antibiotic ointments as well as in antibacterial bandages. There are several case reports of patients with both type IV delayed hypersensitivity (contact dermatitis) and type I anaphylactic reactions to bacitracin ${ }^{38-40}$; patch testers should be aware of this rare association. Bacitracin was positive in $5.5 \%$ of patch tested patients in the 2017 2018 North American Contact Dermatitis Group data cycle, ${ }^{37}$ and as with neomycin, bacitracin also is commonly patch tested in most screening patch test series.

Polymyxin-Polymyxin is a polypeptide topical antibiotic that is used to treat superficial wound infections and can be used in combination with neomycin and/or bacitracin. Historically, it is a less common antibiotic allergen; however, it is now frequently included in comprehensive patch test series, as the frequency of positive reactions seems to be increasing, probably due to polysensitization with neomycin and bacitracin.

Nystatin-Nystatin is an antifungal that binds to ergosterol and disrupts the cell wall. Cases exist of ACD to topical nystatin as well as systemic ACD from oral exposure, though both are quite rare. Authors have surmised that the overall low rates of ACD may be due to poor skin absorption of nystatin, which also can confound patch testing. ${ }^{41,42}$ For patients with suspected ACD to nystatin, repeat open application testing also can be performed to confirm allergy.

Imidazole Antifungals-Similar to nystatins, imidazole antifungals also work by disrupting the fungal cell wall. Imidazole antifungal preparations that have been reported to cause ACD include clotrimazole, miconazole, econazole, and isoconazole, and although crossreactivity patterns have been described, they are not always reproducible with patch testing. ${ }^{43}$ In one reported case, tioconazole found in an antifungal nail lacquer triggered $\mathrm{ACD}$ involving not only the fingers and toes but also the trunk.$^{44}$ Erythema multiforme-like reactions also have been described from topical use. ${ }^{45}$ Commercial patch test preparations of the most common imidazole allergens do exist. Nonimidazole antifungals remain a safe option for allergic patients.

\section{Antihistamines}

Antihistamines, or H1-receptor antagonists, are marketed to be applied topically for relief of pruritus associated with allergic cutaneous reactions. Ironically, they are known 
to be potent sensitizers themselves. There are 6 main chemical classes of antihistamines: phenothiazines, ethylenediamines, ethanolamines, alkylamines, piperazines, and piperidines. Goossens and Linsen $^{46}$ patch tested 12,460 patients from 1978 to 1997 and found the most positive reactions to promethazine (phenothiazine) $(n=12)$, followed by diphenhydramine (ethanolamine) $(n=8)$ and clemizole (benzimidazole) $(n=6)$. The authors also noted cross-reactions between diphenhydramine derivatives and between promethazine and chlorpromazine. ${ }^{46}$

Doxepin is a tricyclic antidepressant with antihistamine activity and is a well-documented sensitizer. ${ }^{47-52}$ Taylor et $\mathrm{al}^{47}$ evaluated 97 patients with chronic dermatoses, and patch testing revealed 17 (17.5\%) positive reactions to doxepin cream, 13 (76.5\%) of which were positive reactions to both the commercial cream and the active ingredient. Patch testing using doxepin dilution as low as $0.5 \%$ in petrolatum is sufficient to provoke a strong $(++)$ allergic reaction. ${ }^{50,51}$ Early-onset $\mathrm{ACD}$ following the use of doxepin cream suggests the possibility of prior sensitization, perhaps with a structurally similar phenothiazine drug. ${ }^{51}$ A keen suspicion for ACD in patients using doxepin cream for longer than the recommended duration can help make the diagnosis. ${ }^{49,52}$

\section{Topical Analgesics}

Nonsteroidal Anti-inflammatory Drugs-Ketoprofen is one of the most frequent culprits of photoallergic contact dermatitis. Pruritic, papulovesicular, and bullous lesions typically develop acutely weeks after exposure. Prolonged photosensitivity is common and can last years after discontinuation of the nonsteroidal anti-inflammatory drug. ${ }^{53}$ Cases of cross-reactions and co-sensitization to structurally similar substances have been reported, including to benzophenone-related chemicals in sunscreen and aldehyde groups in fragrance mix. ${ }^{53,54}$

Diclofenac gel generally is well tolerated in the topical treatment of joint pain and inflammation. In the setting

\section{Recommended Patch Testing Concentrations}

\begin{tabular}{|c|c|}
\hline Topical medicament & Test concentration and vehicle ${ }^{a}$ \\
\hline Retinoic acid (tretinoin) & $0.005 \%$ alc, $0.05 \%$ pet and $0.01 \%-0.02 \%$ alc, $0.1 \%$ pet \\
\hline Benzoyl peroxide & $1 \%$ pet \\
\hline \multicolumn{2}{|l|}{ Brimonidine } \\
\hline \multicolumn{2}{|c|}{ Clindamycin hydrochloride, clindamycin phosphate $1 \%$ water, $1 \%$ pet, $1 \%-20 \%$ water or pet } \\
\hline Metronidazole & $2 \%-5 \%$ pet \\
\hline Neomycin sulfate & $20 \%$ pet \\
\hline Bacitracin & $5 \%-20 \%$ pet \\
\hline Polymyxin B sulfate & $3 \%$ pet \\
\hline Nystatin & $3 \%$ alc, $2 \%$ pet \\
\hline Clotrimazole & $1 \%$ MEK, $1 \%$ pet, $1 \%$ alc, $5 \%$ pet \\
\hline Miconazole nitrate & $2 \%$ alc, $1 \%$ alc, $2 \%$ pet \\
\hline Econazole nitrate & $2 \%$ alc, $1 \%$ alc, $1 \%$ pet \\
\hline Isoconazole nitrate & $1 \%-2 \%$ alc, $1 \%$ pet \\
\hline Promethazine hydrochloride & $1 \%-10 \%$ pet, $2 \%$ pet \\
\hline Diphenhydramine hydrochloride & $1 \%$ pet, $2 \%$ pet \\
\hline Doxepin & $0.5 \%$ and $1 \%$ pet, $5 \%$ pet \\
\hline Ketoprofen & $5 \%$ pet, $1 \%$ pet, $2.5 \%-5 \%$ pet \\
\hline Diclofenac & $2.5 \%$ pet, $2.5 \%-5 \%$ pet, $1 \%$ pet \\
\hline Bufexamac & $5 \%$ pet, $1 \%$ pet \\
\hline Carprofen & $10 \%$ pet, $5 \%$ pet \\
\hline Cyclobenzaprine & $1 \%-2 \%$ pet $^{64}$ \\
\hline Amitriptyline & $5 \%$ pet \\
\hline
\end{tabular}

Abbreviations: alc, alcohol; pet, petrolatum; MEK, methyl ethyl ketone.

apatch testing recommendations were derived from deGroot ${ }^{65}$ unless otherwise indicated. 
of $A C D$, patients typically present with dermatitis localized to the area of application. ${ }^{55}$ Immediate cessation and avoidance of topical diclofenac are crucial components of management. Although systemic contact dermatitis has been reported with oral diclofenac use, ${ }^{56}$ a recent report suggested that oral diclofenac may be well tolerated for some patients with topical ACD. ${ }^{57}$

Publications on bufexamac-induced ACD mainly consist of international reports, as this medication has been discontinued in the United States. Bufexamac is a highly sensitizing agent that can lead to severe polymorphic eruptions requiring treatment with prednisolone and even hospitalization. ${ }^{58}$ In one Australian case report, a mother developed an edematous, erythematous, papulovesicular eruption on the breast while breastfeeding her baby, who was being treated with bufexamac cream 5\% for infantile eczema. ${ }^{59}$ Carprofen-induced photoallergic contact dermatitis is associated with occupational exposure in pharmaceutical workers. ${ }^{60,61} \mathrm{~A}$ few case reports on other nonsteroidal anti-inflammatory drugs, including etofenamate and aceclofenac, have been published. ${ }^{62,63}$

Compounded Medications - Compounded topical analgesics, which help to control pain via multiple combined effects, have gained increasing popularity in the management of chronic neuropathic pain disorders. Only a few recent retrospective studies assessing the efficacy and safety of these medications have mentioned suspected allergic cutaneous reactions. ${ }^{62,63}$ In 2015, Turrentine et al ${ }^{64}$ reported a case of ACD to cyclobenzaprine in a compound containing ketamine $10 \%$, diclofenac $5 \%$, baclofen $2 \%$, bupivacaine $1 \%$, cyclobenzaprine $2 \%$, gabapentin $6 \%$, ibuprofen $3 \%$, and pentoxifylline $3 \%$ in a proprietary cream base. When patients present with suspected ACD to a compounded pain medication, obtaining individual components for patch testing is key to determining the allergic ingredient(s). We suspect that we will see a rise in reports of ACD as these topical compounds become readily adopted in clinical practices.

\section{Patch Testing for Diagnosis}

When patients present with symptoms concerning for ACD to medicaments, the astute clinician should promptly stop the suspected topical medication and consider patch testing. For common allergens such as neomycin, bacitracin, or ethylenediamine, commercial patch test preparations exist and should be used; however, for drugs that do not have a commercial patch test preparation, the patient's product can be applied as is, keeping in mind that certain preparations (such as retinoids) can cause irritant patch test reactions, which may confound the reading. Alternatively, individual ingredients in the medication's formulation can be requested from the manufacturer or a compounding pharmacy for targeted testing. Suggested concentrations for patch testing based on the literature and expert reference are listed in the Table. The authors (M.R., A.R.A.) frequently rely on an expert reference ${ }^{66}$ to determine ideal concentrations for patch testing. Referral to a specialized patch test clinic may be appropriate.

\section{Final Interpretation}

Although their intent is to heal, topical medicaments also can be a source of ACD. The astute clinician should consider ACD when topicals either no longer seem to help the patient or trigger new-onset dermatitis. Patch testing directly with the culprit medicament, or individual medication ingredients when needed, can lead to the diagnosis, though caution is advised. Stay tuned for part 2 of this series in which we will discuss ACD to topical steroids, immunomodulators, and anesthetic medications.

\section{REFERENCES}

1. Davis MD. Unusual patterns in contact dermatitis: medicaments. Dermatol Clin. 2009;27:289-297, vi. doi:10.1016/j.det.2009.05.003

2. Gilissen L, Goossens A. Frequency and trends of contact allergy to and iatrogenic contact dermatitis caused by topical drugs over a 25-year period. Contact Dermatitis. 2016;75:290-302. doi:10.1111/cod.12621

3. Balato N, Patruno C, Lembo G, et al. Allergic contact dermatitis from retinoic acid. Contact Dermatitis. 1995;32:51. doi:10.1111/j.1600-0536.1995.tb00846.x

4. Berg JE, Bowman JP, Saenz AB. Cumulative irritation potential and contact sensitization potential of tazarotene foam $0.1 \%$ in 2 phase 1 patch studies. Cutis. 2012;90:206-211.

5. Numata T, Jo R, Kobayashi $Y$, et al. Allergic contact dermatitis caused by adapalene. Contact Dermatitis. 2015;73:187-188. doi:10.1111/cod.12410

6. Anderson A, Gebauer K. Periorbital allergic contact dermatitis resulting from topical retinoic acid use. Australas J Dermatol. 2014;55:152-153. doi:10.1111/ajd.12041

7. Blondeel A. Contact allergy to vitamin A. Contact Dermatitis. 1984; 11:191-192. doi:10.1111/j.1600-0536.1984.tb00976.x

8. Manzano D, Aguirre A, Gardeazabal J, et al. Allergic contact dermatitis from tocopheryl acetate (vitamin E) and retinol palmitate (vitamin A) in a moisturizing cream. Contact Dermatitis. 1994;31:324. doi:10.1111/j.1600-0536.1994.tb02030.x

9. Heidenheim M, Jemec GB. Occupational allergic contact dermatitis from vitamin A acetate. Contact Dermatitis. 1995;33:439. doi:10.1111/j.1600-0536.1995.tb02091.x

10. Kim C, Craiglow BG, Watsky KL, et al. Allergic contact dermatitis to benzoyl peroxide resemblingimpetigo.Pediatr Dermatol.2015;32:E161-E162. doi:10.1111/pde.12585

11. Sandre M, Skotnicki-Grant S. A case of a paediatric patient with allergic contact dermatitis to benzoyl peroxide. J Cutan Med Surg. 2018;22:226-228. doi:10.1177/1203475417733462

12. Corazza M, Amendolagine G, Musmeci D, et al. Sometimes even Dr Google is wrong: an unusual contact dermatitis caused by benzoyl peroxide. Contact Dermatitis. 2018;79:380-381. doi:10.1111/cod.13086

13. Adelman M, Mohammad T, Kerr H. Allergic contact dermatitis due to benzoyl peroxide from an unlikely source. Dermatitis. 2019;30:230-231. doi:10.1097/DER.0000000000000470

14. Gatica-Ortega ME, Pastor-Nieto MA. Allergic contact dermatitis to Glycyrrhiza inflata root extract in an anti-acne cosmetic product [published online April 28, 2021]. Contact Dermatitis. doi:10.1111/cod.13872

15. Ockenfels HM, Uter W, Lessmann H, et al. Patch testing with benzoyl peroxide: reaction profile and interpretation of positive patch test reactions. Contact Dermatitis. 2009;61:209-216. doi:10.1111/j.1600-0536.2009.01603.x

16. Sodhi PK, Verma L, Ratan J. Dermatological side effects of brimonidine: a report of three cases. J Dermatol. 2003;30:697-700. doi:10.1111/j.1346-8138.2003.tb00461.x

17. Swanson LA, Warshaw EM. Allergic contact dermatitis to topical brimonidine tartrate gel $0.33 \%$ for treatment of rosacea. J Am Acad Dermatol. 2014;71:832-833. doi:10.1016/j.jaad.2014.05.073

18. Bangsgaard N, Fischer LA, Zachariae C. Sensitization to and allergic contact dermatitis caused by Mirvaso ${ }^{(®)}$ (brimonidine tartrate) for treatment of rosacea—2 cases. Contact Dermatitis. 2016;74:378-379. doi:10.1111/cod.12547

19. Ringuet J, Houle MC. Case report: allergic contact dermatitis to topical brimonidine demonstrated with patch testing: insights on evaluation of brimonidine sensitization. J Cutan Med Surg. 2018;22:636-638. doi:10.1177/1203475418789020 
20. Cookson H, McFadden J, White J, et al. Allergic contact dermatitis caused by Mirvaso $®$, brimonidine tartrate gel $0.33 \%$, a new topical treatment for rosaceal erythema. Contact Dermatitis. 2015;73:366-367. doi:10.1111/cod.12476

21. Rajagopalan A, Rajagopalan B. Allergic contact dermatitis to topical brimonidine. Australas J Dermatol. 2015;56:235. doi:10.1111/ajd.12299

22. Veraldi S, Brena M, Barbareschi M. Allergic contact dermatitis caused by topical antiacne drugs. Expert Rev Clin Pharmacol. 2015;8:377-381. doi:10.1586/17512433.2015.1046839

23. Vejlstrup E, Menné T. Contact dermatitis from clindamycin. Contact Dermatitis. 1995;32:110. doi:10.1111/j.1600-0536.1995.tb00759.x

24. García R, Galindo PA, Feo F, et al. Delayed allergic reactions to amoxycillin and clindamycin. Contact Dermatitis. 1996;35:116-117. doi:10.1111/j.1600-0536.1996.tb02312.x

25. Muñoz D, Del Pozo MD, Audicana M, et al. Erythema-multiformelike eruption from antibiotics of 3 different groups. Contact Dermatitis. 1996;34:227-228. doi:10.1111/j.1600-0536.1996.tb02187.x

26. Romita P, Ettorre G, Corazza M, et al. Allergic contact dermatitis caused by clindamycin mimicking'retinoid flare.' Contact Dermatitis. 2017;77:181-182. doi:10.1111/cod.12784

27. Veraldi S, Guanziroli E, Ferrucci S, et al. Allergic contact dermatitis caused by clindamycin. Contact Dermatitis. 2019;80:68-69. doi:10.1111/cod.13133

28. Voller LM, Kullberg SA, Warshaw EM. Axillary allergic contact dermatitis to topical clindamycin. Contact Dermatitis. 2020;82:313-314. doi:10.1111 /cod.13465

29. de Kort WJ, de Groot AC. Clindamycin allergy presenting as rosacea. Contact Dermatitis. 1989;20:72-73. doi:10.1111/j.1600-0536.1989.tb03108.x

30. Vincenzi C, Lucente P, Ricci C, et al. Facial contact dermatitis due to metronidazole. Contact Dermatitis. 1997;36:116-117. doi:10.1111/j.1600-0536.1997. tb00434.x

31. Wolf $\mathrm{R}$, Orion E, Matz H. Co-existing sensitivity to metronidazole and isothiazolinone. Clin Exp Dermatol. 2003;28:506-507. doi:10.1046 /j.1365-2230.2003.01364.x

32. Madsen JT, Thormann J, Kerre S, et al. Allergic contact dermatitis to topical metronidazole—3 cases. Contact Dermatitis. 2007;56:364-366. doi:10.1111 j.1600-0536.2006.01064.x

33. Fernández-Jorge B, Goday Buján J, Fernández-Torres R, et al. Concomitant allergic contact dermatitis from diphenhydramine and metronidazole. Contact Dermatitis. 2008;59:115-116. doi:10.1111/j.1600-0536.2008.01332.x

34. Madsen JT, Lorentzen HF, Paulsen E. Contact sensitization to metronidazole from possible occupational exposure. Contact Dermatitis. 2009; 60:117-118. doi:10.1111/j.1600-0536.2008.01490.x

35. Beutner KR, Lemke S, Calvarese B. A look at the safety of metronidazole $1 \%$ gel: cumulative irritation, contact sensitization, phototoxicity, and photoallergy potential. Cutis. 2006;77(4 suppl):12-17.

36. Jappe U, Schäfer T, Schnuch A, et al. Contact allergy in patients with rosacea: a clinic-based, prospective epidemiological study. J Eur Acad Dermatol Venereol. 2008;22:1208-1214. doi:10.1111/j.1468-3083.2008.02778.x

37. DeKoven JG, Silverberg JI, Warshaw EM, et al. North American Contact Dermatitis Group Patch Test Results: 2017-2018. Dermatitis. 2021;32:111-123. doi:10.1097/DER.0000000000000729

38. Comaish JS, Cunliffe WJ. Absorption of drugs from varicose ulcers: a cause of anaphylaxis. Br J Clin Pract. 1967;21:97-98.

39. Roupe G, Strannegård O. Anaphylactic shock elicited by topical administration of bacitracin. Arch Dermatol. 1969;100:450-452.

40. Farley M, Pak H, CarregalV, et al. Anaphylaxis to topically applied bacitracin. Am J Contact Dermat. 1995;6:28-31.

41. Barranco R, Tornero $\mathrm{P}$, de Barrio $\mathrm{M}$, et al. Type IV hypersensitivity to oral nystatin. Contact Dermatitis. 2001;45:60. doi:10.1034/j.1600 -0536.2001.045001060x

42. Cooper SM, Shaw S. Contact allergy to nystatin: an unusual allergen. Contact Dermatitis. 1999;41:120. doi:10.1111/j.1600-0536.1999.tb06254.x

43. Dooms-Goossens A, Matura M, Drieghe J, et al. Contact allergy to imidazoles used as antimycotic agents. Contact Dermatitis. 1995;33:73-77. doi:10.1111/j.1600-0536.1995.tb00504.x

44. Pérez-Mesonero R, Schneller-Pavelescu L, Ochando-Ibernón G, et al. Is tioconazole contact dermatitis still a concern? bringing allergic contact dermatitis caused by topical tioconazole back into the spotlight. Contact Dermatitis. 2019;80:168-169.
45. Tang MM, Corti MA, Stirnimann R, et al. Severe cutaneous allergic reactions following topical antifungal therapy. Contact Dermatitis. 2013;68:56-57.

46. Goossens A, Linsen G. Contact allergy to antihistamines is not common. Contact Dermatitis. 1998;39:38. doi:10.1111/j.1600-0536.1998. tb05817.x

47. Taylor JS, Praditsuwan P, Handel D, et al. Allergic contact dermatitis from doxepin cream. one-year patch test clinic experience. Arch Dermatol. 1996;132:515-518.

48. Bilbao I, Aguirre A, Vicente JM, et al. Allergic contact dermatitis due to 5\% doxepin cream. Contact Dermatitis. 1996;35:254-255. doi:10.1111/j.1600-0536.1996.tb02374.x

49. Shelley WB, Shelley ED, Talanin NY. Self-potentiating allergic contact dermatitis caused by doxepin hydrochloride cream. J Am Acad Dermatol. 1996;34:143-144. doi:10.1016/s0190-9622(96)90864-6

50. Wakelin SH, Rycroft RJ. Allergic contact dermatitis from doxepin. Contact Dermatitis. 1999;40:214. doi:10.1111/j.1600-0536.1999.tb06037.x

51. Horn HM, Tidman MJ, Aldridge RD. Allergic contact dermatitis due to doxepin cream in a patient with dystrophic epidermolysis bullosa. Contact Dermatitis. 2001;45;115. doi:10.1034/j.1600 $-0536.2001 .045002115 . x$

52. Bonnel RA, La Grenade L, Karwoski CB, et al. Allergic contact dermatitis from topical doxepin: Food and Drug Administration's postmarketing surveillance experience. J Am Acad Dermatol. 2003;48:294-296. doi:10.1067/mjd.2003.46

53. Devleeschouwer V, Roelandts R, Garmyn M, et al. Allergic and photoallergic contact dermatitis from ketoprofen: results of (photo) patch testing and follow-up of 42 patients. Contact Dermatitis. 2008;58:159-166. doi:10.1111/j.1600-0536.2007.01296.x

54. Foti C, Bonamonte D, Conserva A, et al. Allergic and photoallergic contact dermatitis from ketoprofen: evaluation of crossreactivities by a combination of photopatch testing and computerized conformational analysis. Curr Pharm Des. 2008;14:2833-2839. doi:10.2174/138161208786369696

55. Gulin SJ, Chiriac A. Diclofenac-induced allergic contact dermatitis: a series of four patients. Drug Saf Case Rep. 2016;3:15. doi:10.1007/s40800 -016-0039-3

56. Lakshmi C, Srinivas CR. Systemic (allergic) contact dermatitis to diclofenac. Indian J Dermatol Venereol Leprol. 2011;77:536. doi:10.4103/0378 $-6323.82424$

57. Beutner C, Forkel S, Kreipe K, et al. Contact allergy to topical diclofenac with systemic tolerance [published online August 22, 2021]. Contact Dermatitis. doi:10.1111/cod.13961

58. Pan Y, Nixon R. Allergic contact dermatitis to topical preparations of bufexamac. Australas J Dermatol. 2012;53:207-210. doi:10.1111 jj.1440-0960.2012.00876.x

59. Nakada T, Matsuzawa Y. Allergic contact dermatitis syndrome from bufexamac for nursing infant. Dermatitis. 2012;23:185-186. doi:10.1097 /DER.0b013e318260d774

60. Kerr AC, Muller F, Ferguson J, et al. Occupational carprofen photoallergic contact dermatitis. Br J Dermatol. 2008;159:1303-1308. doi:10.1111/j 1365-2133.2008.08847.x

61. Kiely C, Murphy G. Photoallergic contact dermatitis caused by occupational exposure to the canine non-steroidal anti-inflammatory drug carprofen. Contact Dermatitis. 2010;63:364-365. doi:10.1111 jj.1600-0536.2010.01820.x

62. Somberg J, Molnar J. Retrospective evaluation on the analgesic activities of 2 compounded topical creams and voltaren gel in chronic noncancer pain. Am J Ther. 2015;22:342-349. doi:10.1097 /MJT.0000000000000275

63. Lee HG, Grossman SK, Valdes-Rodriguez R, et al. Topical ketamineamitriptyline-lidocaine for chronic pruritus: a retrospective study assessing efficacy and tolerability. J Am Acad Dermatol. 2017;76:760-761. doi:10.1016/j.jaad.2016.10.030

64. Turrentine JE, Marrazzo G, Cruz PD Jr. Novel use of patch testing in the first report of allergic contact dermatitis to cyclobenzaprine. Dermatitis. 2015;26:60-61. doi:10.1097/DER.0000000000000099

65. de Groot A. Patch Testing. 3rd ed. acdegroot publishing; 2008.

66. de Groot A. Patch Testing. 4th ed. acdegroot publishing; 2018. 